\title{
Molecular mechanisms in vascular injury induced by hypertension: Expression and role of microRNA-34a
}

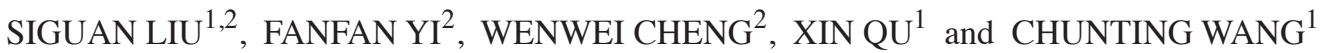 \\ ${ }^{1}$ Department of Critical Care Medicine, Shandong Provincial Hospital Affiliated to Shandong University, Jinan, Shandong \\ 250101; ${ }^{2}$ Emergency Department, Zaozhuang Municipal Hospital, Zaozhuang, Shandong 277101, P.R. China
}

Received May 17, 2016; Accepted April 7, 2017

DOI: $10.3892 / \mathrm{etm} .2017 .5216$

\begin{abstract}
The aim of the present study was to investigate the expression and function of microRNA (miR)-34a in patients with primary hypertension. The expression of miR-34a was measured in the peripheral blood of 50 patients with primary hypertension and 28 normal controls by reverse transcription quantitative polymerase chain reaction. In addition, human umbilical vein endothelial cells (HUVECs) were transfected with an miR-34a inhibitor to suppress the expression of miR-34a, and the proliferation, migration and cell cycle distribution of HUVECs were measured by Cell Counting Kit-8, Transwell and flow cytometry assays. The target of miR-34a was also predicted by bioinformatics analysis and verified by a dual-luciferase reporter gene assay and western blot analysis. miR-34a was significantly upregulated in the peripheral blood of patients with hypertension when compared with controls $(\mathrm{P}<0.05)$, and upregulation of miR-34a was associated with a higher clinical stage of hypertension (phase III; $\mathrm{P}<0.05)$. In vitro experiments demonstrated that inhibition of miR-34a promoted the proliferation, migration and G1/S transition of HUVECs, relative to scramble-miR controls $(\mathrm{P}<0.05)$. Furthermore, transforming growth factor $\beta$-induced factor homeobox 2 (TIGF2) was predicted and verified to be a direct target of miR-34a. Collectively, these data suggested that miR-34a was upregulated in the peripheral blood of patients with hypertension, and that upregulated miR-34a may promote vascular endothelial injury by targeting TIGF2.
\end{abstract}

\section{Introduction}

Primary hypertension is a common chronic disease and typically causes other health complications, including coronary

Correspondence to: Professor Chunting Wang, Department of Critical Care Medicine, Shandong Provincial Hospital Affiliated to Shandong University, 9677 Jingshi Road, Jinan, Shandong 250101, P.R. China

E-mail:wtr333@126.com

*Contributed equally

Key words: hypertension, vascular endothelial injury, microRNA-34a, transforming growth factor $\beta$-induced factor homeobox 2 artery disease, stroke, renal failure and heart failure, and poses a great threat to human health (1-3). At present, 300 million patients with hypertension, and 10 million patients are diagnosed annually in China (4). However, the mechanisms underlying the development of primary hypertension remain unclear. Over the last decade, the incidence of primary hypertension and percentage of young patients with primary hypertension in China has increased (5), which has drawn the attention of a number of researchers.

Vascular injury, including vascular endothelial injury, smooth muscle cell proliferation and vascular remodeling, is the basic pathological change of hypertension diseases (6-8). A previous study demonstrated that vascular endothelial injury is important in the development of hypertension (9). Hypertension induces vascular endothelial dysfunction, disrupts the balance of nitric oxide (NO) and secretion of endothelin from vascular endothelial cells and increases the contraction of arteries, leading to pulmonary arterial hypertension characterized by a persistent increase in pulmonary artery pressure (10). In a hypertensive microenvironment, the apoptosis of microvascular endothelial cells is increased, resulting in a reduction of pathways in the capillary network that allow gas exchange between tissues and elevation of peripheral circulation pressure, which ultimately leads to high blood pressure (11). However, the molecular mechanism underlying hypertension-induced vascular endothelial injury is not well understood and warrants further study.

MicroRNAs (miRNA) are a class of highly conserved, non-coding RNAs of 18-22 nucleotides in length, which predominantly serve as translational repressors by binding to complementary sequences in the $3^{\prime}$ untranslated region (UTR) of their target mRNAs (12). A recent study demonstrated that the miRNA expression pattern in the peripheral blood of patients with hypertension was altered, which may be used clinically for the early diagnosis of hypertension and the prognosis of hypertension-induced complications (13). In addition, miRNAs may regulate hypertension-induced complications and vascular endothelial injury (14). It has been demonstrated that miR-34a is ectopically expressed in the peripheral blood of patients with hypertension (15), suggesting that this miRNA is associated with the development of hypertension. However, the specific mechanism remains unclear. The current study aimed to investigate the expression of miRNA (miR)-34a in the peripheral blood of patients with hypertension and its role in regulating vascular endothelial injury. 


\section{Materials and methods}

Clinical data and peripheral blood collection. Peripheral blood from 50 patients with primary hypertension and 28 healthy volunteers was collected between December 2013 and October 2014 from the Shandong Provincial Hospital Affiliated to Shandong University (Jinan, China). The blood samples were stored on ice for $<20 \mathrm{~min}$ until further processing. A total of 24 male and 26 female patients were included in the study. The average age of patients was 61.5 years (ranging between 51 and 82) and the median age was 62 years. Patients with a duration of hypertension $>5$ years were enrolled. Patients with combination of other chronic underlying diseases (such as diabetes) or long history of medication were excluded. Written informed consent was obtained from all patients prior to the study, and the study was approved by the Ethics Review Board of Shandong Provincial Hospital Affiliated to Shandong University.

Patients were divided into three phases, based on the phase of hypertension observed (16). Phase I $(n=21)$, systolic pressure is $140-159 \mathrm{mmHg}$ or diastolic blood pressure is $90-99 \mathrm{mmHg}$; Phase II $(\mathrm{n}=16)$, systolic pressure is $160-179 \mathrm{mmHg}$ or diastolic blood pressure is $100-109 \mathrm{mmHg}$; Phase III $(\mathrm{n}=13)$, systolic pressure $>180 \mathrm{mmHg}$ or diastolic blood pressure $>100 \mathrm{mmHg}$.

miRNA transfection of human umbilical vein endothelial cells (HUVECS).HUVECs were purchased from ScienCell Research Laboratories, Inc. (Carlsbad, CA, USA; cat. no. 8000) and maintained at $37^{\circ} \mathrm{C}$ in Dulbecco's modified Eagle's medium (DMEM; Gibco; Thermo Fisher Scientific, Inc., Waltham, MA, USA) supplemented with $10 \%$ fetal bovine serum (FBS; Thermo Fisher Scientific, Inc.). Cells were seeded into 24-well plates at a concentration of $1 \times 10^{5}$ cells/well. When the HUVECs reached $70-90 \%$ confluence, the cells were transfected with $2.5 \mu 1 \mathrm{miR}-34 \mathrm{a}$ inhibitor (targeting sequence; 5'-CAATCA GCAAGTATACTGCCCT-3', $25 \mathrm{pmol} / \mu \mathrm{l}$ ) or scramble-miR (negative control, NC; both from Guangzhou RiboBio Co., Ltd., Guangzhou, China) using Lipofectamine ${ }^{\circledR} 2000$ (Thermo Fisher Scientific, Inc.), according to the manufacturer's instructions. Cells were cultured $48 \mathrm{~h}$ post-transfection at $37^{\circ} \mathrm{C}$ with $5 \% \mathrm{CO}_{2}$ until collection for subsequent experiments.

$R N A$ extraction and reverse transcription quantitative polymerase chain reaction ( $R T-q P C R)$ assay. Total RNA was extracted from cells using TRIzol ${ }^{\circledR}$ isolation reagent (Thermo Fisher Scientific, Inc.) according to the manufacturer's instructions. Following verification of RNA integrity by gel electrophoresis and quantification using a UV spectrophotometer at 260 and $280 \mathrm{~nm}, 0.5 \mu \mathrm{g}$ total RNA was reverse transcribed using an miScript II RT kit (Qiagen, Hilden, Germany). The expression of small nuclear U6 was used as an internal control. qPCR was performed using a KAPA SYBR $^{\circledR}$ FAST qPCR kit (Kapa Biosystems, Inc., Wilmington, MA, USA) at $95^{\circ} \mathrm{C}$ for $10 \mathrm{~min}$ and 40 cycles of $95^{\circ} \mathrm{C}$ for $1 \mathrm{~min}$ and $60^{\circ} \mathrm{C}$ for $30 \mathrm{sec}$. The forward primer used for miR-34a amplification was 5'-CAGTGTCTTAGCTGGTTG-3', and the reverse primer was provided within the kit. Primers used for amplification of U6 were as follows: Forward, 5'-CTCGCT TCGGCAGCACA-3' and reverse, 5'-AACGCTTCACGAATT
TGCGT-3'. Relative expression levels were determined using the $\Delta \Delta \mathrm{C}_{\mathrm{q}}$ method (17).

Cell Counting Kit-8 (CCK-8) assay. To evaluate the effect of miR-34a on the proliferation of HUVECs, cells were collected $48 \mathrm{~h}$ after transfection and washed twice with phosphate-buffered saline (PBS). The cells were then suspended in fresh DMEM containing 10\% CCK-8 solution (Biyuntian Biotech Co., Ltd, Shanghai, China) and incubated for $1 \mathrm{~h}$ at $37^{\circ} \mathrm{C}$. The absorbance of each well was measured with an ELx800 Microplate Reader (Biotek Instruments, Inc., Winooski, VT, USA) set at $450 \mathrm{~nm}$.

Flow cytometric analysis of cell apoptosis. At $48 \mathrm{~h}$ after transfection, $10^{6}$ cells were harvested $48 \mathrm{~h}$ after transfection by $0.25 \%$ trypsin digestion at $37^{\circ} \mathrm{C}$ for $3 \mathrm{~min}$, washed twice with cold PBS and stained with an Annexin V-fluorescein isothiocyanate (FITC) apoptosis detection kit I (BD Biosciences, San Jose, CA, USA) according to the manufacturer's instructions. Cells were immediately analyzed by BD FACSVerse ${ }^{\mathrm{TM}}$ flow cytometer (BD Biosciences) with Modfit software (version 1.0.1; Verity Software House, Inc., Topsham, ME, USA).

Flow cytometry analysis of cell cycle. At $48 \mathrm{~h}$ after transfection, $1 \times 10^{6}$ cells were washed twice with cold PBS and stained with a BD Cycletest Plus DNA Reagent (cat. no. 340242; BD Biosciences), according to the manufacturer's instructions. Cells were analyzed by flow cytometry using a BD FACSVerse ${ }^{\mathrm{TM}}$ (BD Biosciences) with Modfit software, version 2.0.

Migration assay. A total of $1 \times 10^{5}$ HUVECs in $200 \mu 1$ RPMI-1640 medium (Thermo Fisher Scientific, Inc.) without FBS were seeded into the top chamber of an $8-\mu \mathrm{m}$ pore filter Transwell chamber (Corning Incorporated, Corning, NY, USA) inserted in 24-well plates. RPMI-1640 plus 10\% FBS $(500 \mu \mathrm{l})$ was added to the bottom chamber, and the cells were incubated at $37^{\circ} \mathrm{C}$ and $5 \% \mathrm{CO}_{2}$. After $24 \mathrm{~h}$, the cells that did not pass through the chambers were removed with a cotton swab, while the cells located on the lower side of the chamber were fixed with $4 \%$ paraformaldehyde at room temperature for 10 min, stained with Giemsa and counted using an Olympus BX51/61 microscope (Olympus Corporation, Tokyo, Japan) at a magnification of $x 200$. Migration data were collected by counting the migrated cells in 5 randomly selected fields.

Western blot analysis. Following transfection with miR-34a inhibitor or scramble-miR, cells were collected and resuspended in radioimmunoprecipitation assay lysis buffer with $1 \%$ phenylmethanesulfonyl fluoride (Beyotime Institute of Biotechnology, Beijing, China) at room temperature for $30 \mathrm{~min}$ to extract total protein. Each sample was centrifuged at $12,000 \mathrm{x} \mathrm{g}$ for $10 \mathrm{~min}$ at $4^{\circ} \mathrm{C}$. The enhanced BCA protein assay kit (cat. no. P0009; Beyotime Institute of Biotechnology) including bovine serum albumin as the standard was used to measure the concentration of total protein. A total of $20 \mu \mathrm{g}$ protein per lane was separated by $12 \%$ SDS-PAGE and detected with rabbit anti-TGIF2 antibody (1:1,000; 11522-1-AP) based on results of the bioinformatics prediction and mouse anti-GAPDH antibody $(1: 5,000 ; 60004-1-\mathrm{Ig})$ at $4^{\circ} \mathrm{C}$ overnight. Antibodies were purchased from ProteinTech 
Group, Inc. (Wuhan Sanying Biotechnology, Wuhan, China). Rabbit anti-mouse IgG H\&L conjugated to horseradish peroxidase (HRP; cat. no. ab6728; 1:5,000) and Goat anti-rabbit IgG H\&L conjugated to HRP (cat. no. ab6721; 1:5,000; Abcam, Cambridge, UK) were used as secondary antibodies and incubated at room temperature for $2 \mathrm{~h}$. Immunolabeled bands were detected by BeyoECL Plus (Beyotime, Beijing, China; cat. no. P0018). Each western blot analysis was replicated 3 times.

Bioinformatic prediction. The targets of miR-34a were predicted by Targetscan 7.1 (www.targetscan.org). Species set as human and miR-34a was entered into the microRNA name field and searched.

Dual-luciferase reporter gene assay. According to the results of the bioinformatics prediction, a conservative miR-34a binding sequence complimentary to the $3^{\prime}$ UTR of Tgif 2 mRNA with the smallest $\mathrm{P}$-value $(\mathrm{P}<0.001)$ was selected. Luciferase reporter plasmids were generated by insertion of wild-type (5'-GGG UUUUCUAUGGAUCACUGCCA-3') or mutant binding sequences (5'-GGGUUUUCUAUGGAUAAGUACAA-3') of TGIF2 into the multiple cloning site (SpeI and HindIII) of a pMIR-REPORT ${ }^{\mathrm{TM}}$ luciferase plasmid downstream of the luciferase reporter gene, provided by Hanbio Biotechnology, Co., Ltd. HEK293T cells (105) (ScienCell Research Laboratories, Inc.) were cultured at $37^{\circ} \mathrm{C}$ in Dulbecco's modified Eagle's medium (DMEM; Gibco; Thermo Fisher Scientific, Inc.) supplemented with $10 \%$ FBS (Thermo Fisher Scientific, Inc.) for $24 \mathrm{~h}$. Cell were transfected with $1 \mu \mathrm{g}$ constructed luciferase reporters and $100 \mathrm{nM}$ miR-34a mimics (5'-TGGCAGTGT CTTAGCTGGTTGT-3') or NC RNA (Hanbio Biotechnology Co., Ltd.). A total of $10 \mathrm{ng}$ pMIR-REPORT ${ }^{\mathrm{TM}} \beta$-gal control plasmid (Beyotime Institute of Biotechnology) was transfected as an internal control to determine transfection efficiency. Luminescence was measured $24 \mathrm{~h}$ after transfection using a dual-luciferase detection kit (cat. no. RG027; Beyotime Institute of Biotechnology), according to the manufacturer's instructions. Measurements of luminescence were performed with a luminometer $\left(\right.$ Glomax $^{\circledR} 20 / 20$; Promega Corporation, Madison, WI, USA).

Statistical analysis. The statistical significance of data was determined with paired t-tests using SPSS 16.0 software (SPSS, Inc., Chicago, IL, USA). All data were presented as the mean \pm standard deviation of 3 independent experiments, and $\mathrm{P}<0.05$ was considered to indicate a statistically significant difference.

\section{Results}

miR-34a expression in the peripheral blood of patients with primary hypertension. Initially, the potential dysregulation of miR-34a in patients with hypertension was investigated. Using RT-qPCR analysis, upregulation of miR-34a was identified in hypertensive patients when compared with normal subjects $(\mathrm{P}<0.05$; Fig. 1A). By analyzing miR-34a expression in various groups based on clinical pathological features, it was also observed that miR-34a was significantly upregulated in patients with phase III hypertension when compared with patients presenting with phase I and II hypertension $(\mathrm{P}<0.05$;
Table I. Clinical data of patients categorized into different phases of hypertension.

\begin{tabular}{lccc}
\hline Phase & Number & Mean age, years & Male/Female \\
\hline I & 21 & 54.5 & $8 / 13$ \\
II & 16 & 68.6 & $12 / 4$ \\
III & 13 & 57.4 & $4 / 9$ \\
\hline
\end{tabular}
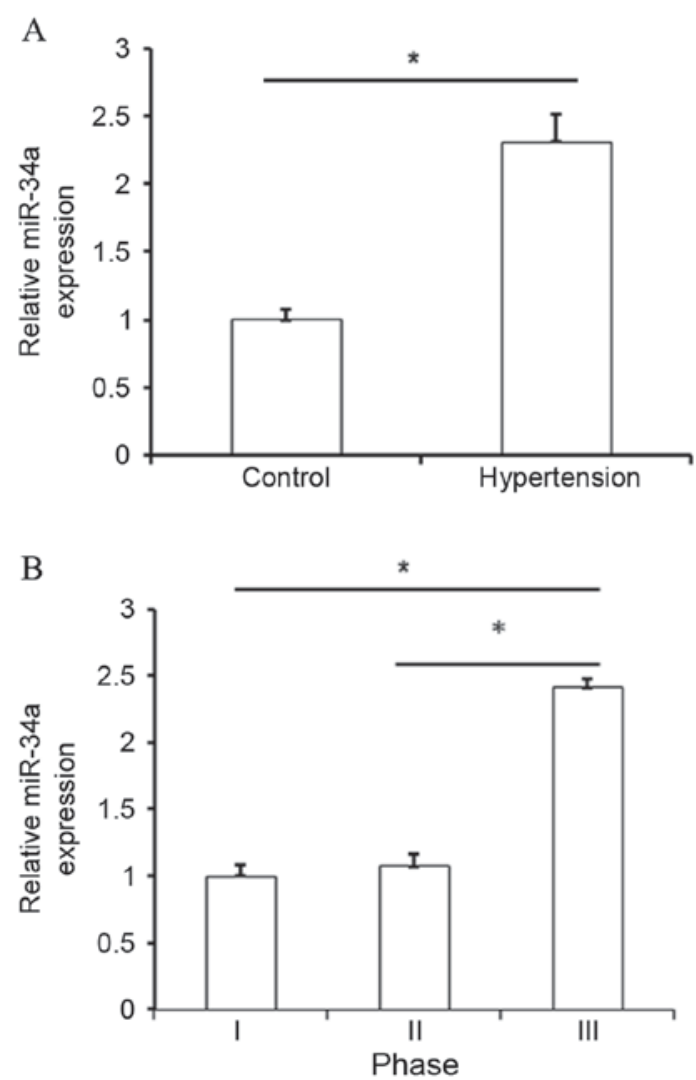

Figure 1. Expression of miR-34a in the peripheral blood of patients with hypertension. (A) Peripheral blood was collected from patients with hypertension and normal subjects, and the expression of miR-34a was assessed by reverse transcription-quantitative polymerase chain reaction. (B) $\mathrm{miR}-34 \mathrm{a}$ expression was also assessed in the peripheral blood of patients with different stages of hypertension. ${ }^{*} \mathrm{P}<0.05$. miR, microRNA.

Fig. 1B and Table I). These results indicated that upregulation of miR-34a was correlated with the development of hypertension.

Effect of miR-34a on the proliferation of HUVECs. A CCK-8 assay demonstrated that transfection with miR-34a inhibitor significantly promoted the proliferation of HUVECs in vitro $(\mathrm{P}<0.05$ vs. NC; Fig. 2). This suggests that increased miR-34a expression in the peripheral blood of patients with hypertension may inhibit the proliferation of vascular endothelial cells.

miR-34a suppresses the migration of HUVECs. The effect of miR-34a on cell migration was subsequently evaluated. A Transwell assay revealed that inhibition of miR-34a expression significantly increased the migration of HUVECs, as demonstrated by an increased number of cells that passed through 


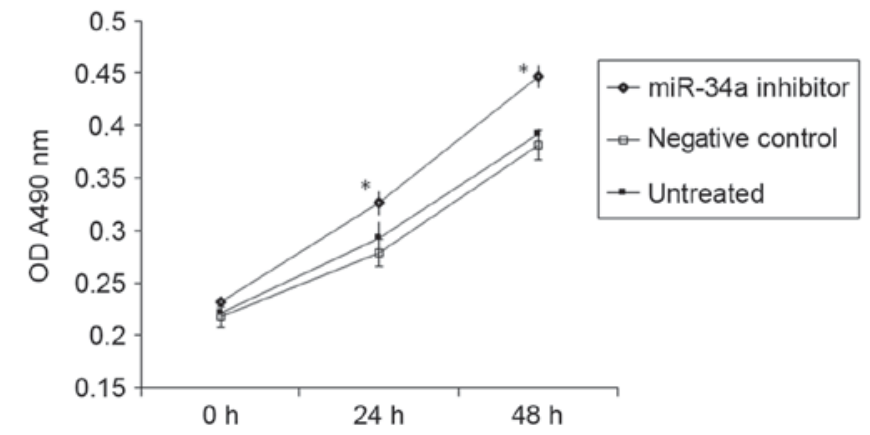

Figure 2. Suppression of miR-34a promotes HUVEC cell proliferation. HUVECs were transfected with miR-34a inhibitor or NC scramble-miR, and cell proliferation was assessed by a Cell Counting Kit -8 assay. ${ }^{~} \mathrm{P}<0.05$ vs. NC. miR, microRNA; HUVECs, human umbilical vein endothelial cells; NC, negative control.
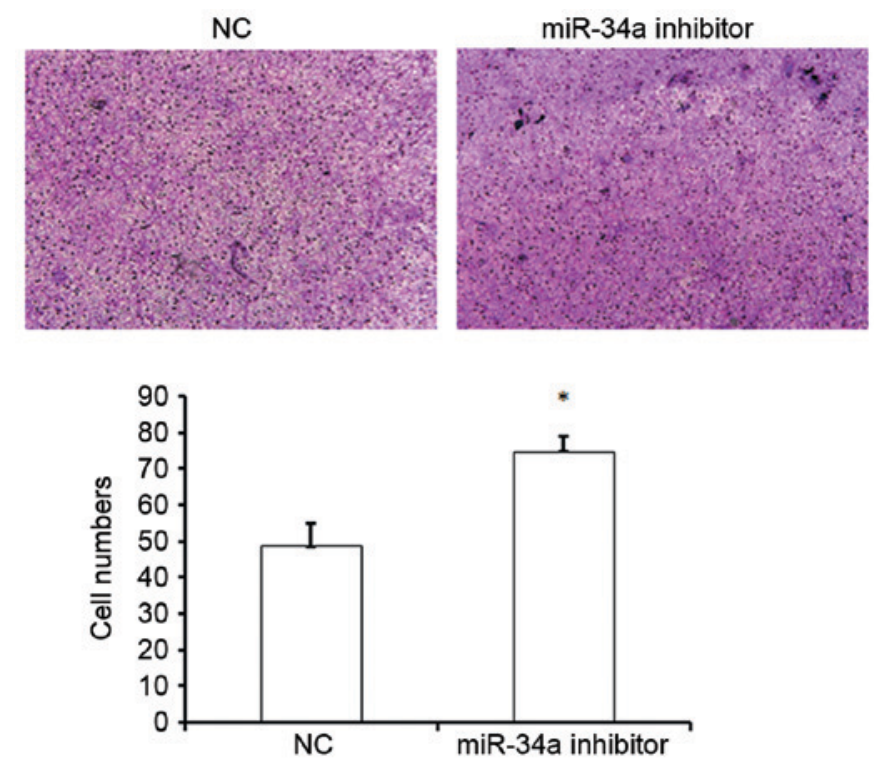

Figure 3. Suppression of miR-34a inhibits HUVEC cell migration. Transwell migration of HUVECs transfected with miR-34a inhibitor or NC scramble-miR. Cells that migrated through the filter into the lower wells were stained with Giemsa (purple; magnification, $\mathrm{x} 200$ ). The number of migrated cells was counted. " $\mathrm{P}<0.05$ vs. NC. miR, microRNA; HUVECs, human umbilical vein endothelial cells; $\mathrm{NC}$, negative control.

the chambers compared with the NC group $(74.5 \pm 4.30$ vs. $48.5 \pm 6.3 ; \mathrm{P}<0.05$; Fig. 3 ). This result suggests that miR-34a may suppress the migration of HUVECs, and thus may inhibit the migration of vascular endothelial cells to sites of injury in vivo, leading to the inhibition of vascular injury repair.

Effect of miR-34a on cell cycle distribution. Following transfection of HUVECs with miR-34a inhibitor, cell cycle distribution was evaluated by flow cytometry. The G1/S transition was significantly promoted in cells transfected with miR-34a inhibitor when compared with NC cells $(\mathrm{P}<0.05$; Fig. 4). This data indicates that miR-34a may inhibit the proliferation and repair of vascular endothelial cells by regulation of the $\mathrm{G} 1 / \mathrm{S}$ transition.

Effect of miR-34a on the apoptosis of HUVECs. To determine the effect of miR-34a on cell apoptosis, HUVECs were
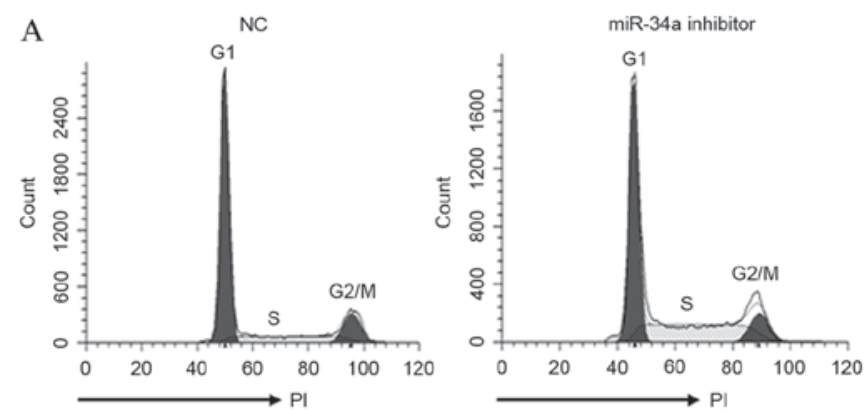

B

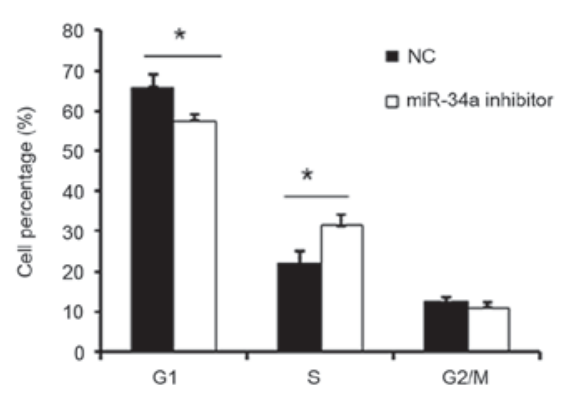

Figure 4. Flow cytometry analysis of cell cycle distribution. (A) Cell cycle distribution was analyzed by flow cytometry $24 \mathrm{~h}$ after transfection of human umbilical vein endothelial cells with an miR-34a inhibitor or NC scramble-miR. (B) The percentages of cells at G1, S and G2/M phase were calculated. ${ }^{*} \mathrm{P}<0.05$. miR, microRNA; PI, propidium iodide; NC, negative control.

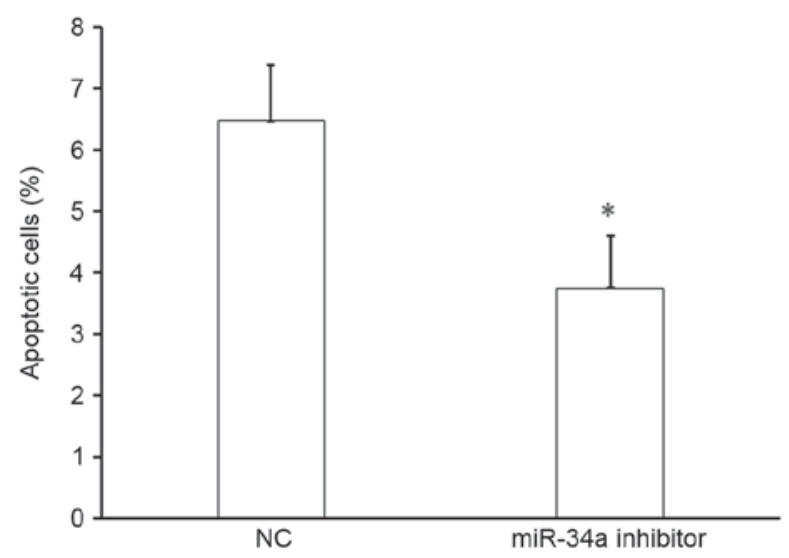

Figure 5. Suppression of miR-34a inhibits HUVEC apoptosis. HUVECs were transfected with miR-34a inhibitor or NC scramble-miR. Cell apoptosis was analyzed by flow cytometry and the percentages of apoptotic cells were calculated. "P<0.05 vs. NC. miR, microRNA; HUVECs, human umbilical vein endothelial cells; $\mathrm{NC}$, negative control.

transfected with miR-34a inhibitor or scramble-miR (NC), stained with Annexin V-FITC and propidium iodide, and analyzed by flow cytometry. There was a significant decrease in the number of apoptotic cells following transfection with miR-34a inhibitor compared with that following NC transfection $(\mathrm{P}<0.05$; Fig. 5$)$. Thus, miR-34a promotes the apoptosis of HUVECs and miR-34a was upregulated in patients with hypertension, suggesting that miR-34a may accelerate vascular endothelial injury.

Tigf2 is a target of miR-34a. Next, the downstream targets of miR-34a were investigated. As predicted by computational 
A

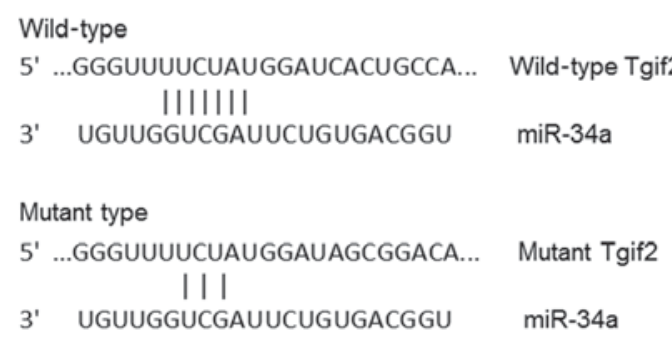

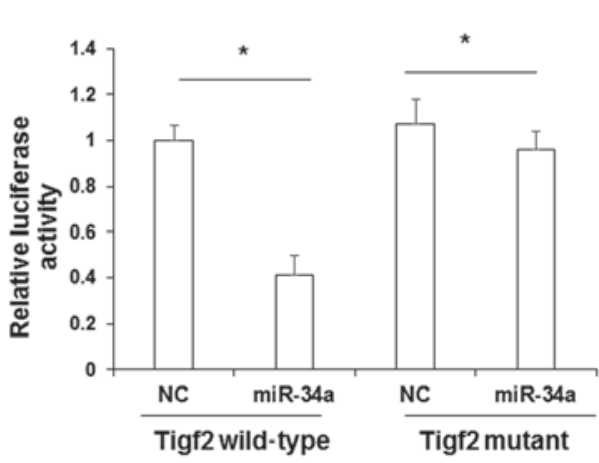

B

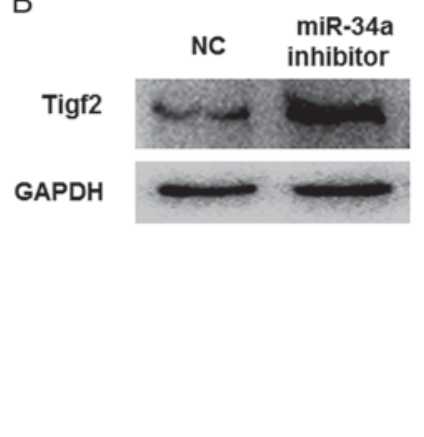

Figure 6. Tigf2 is a target of miR-34a. (A) The results of bioinformatics. Wild-type or mutant Tigf2 3'UTR luciferase reporter constructs were cotransfected with miR-34a mimic or NC scramble-miR, and luciferase activities were assayed $24 \mathrm{~h}$ post-transfection. (B) Human umbilical vein endothelial cells were transfected with an miR-34a inhibitor or NC scramble-miR. Cell lysates were prepared and assessed by western blot analysis to detect the expression of TIGF2 and GAPDH (internal control). $\mathrm{P}<0.05$. Tigf2, transforming growth factor $\beta$-induced factor homeobox 2; miR, microRNA; UTR, untranslated region; NC, negative control.

screening, the 3' UTR of Tigf2 was identified to contain multiple potential binding sites for miR-34a. To determine whether miR-34a directly targeted Tigf2, reporter gene assays were performed. The binding sequence of miR-34a to Tigf2 is presented in Fig. 6A. Cotransfection of a Tigf2 wild-type 3' UTR construct and miR-34a mimic in HEK293T cells lead to a significant reduction in relative luciferase activity $(\mathrm{P}<0.05$ vs. Tigf2 wild-type 3 ' UTR + NC transfectants $)$. By contrast, relative luciferase activity following cotransfection with Tigf2 mutant 3' UTR and miR-34a mimic was not significantly altered when compared with Tigf 2 mutant $3^{\prime}$ UTR + NC transfectants. To verify that TIGF2 was a target of miR-34a, a western blot analysis was performed. It was observed that the protein expression of TIGF2 was markedly upregulated following transfection with miR-34a inhibitor when compared with NC cells (Fig. 6B). These data indicate that the biological function of miR-34a may be correlated with the level of TIGF2 expression.

\section{Discussion}

In the present study, miR-34a was significantly upregulated in the peripheral blood of patients with hypertension, and its expression was correlated with the clinical phase of hypertension. In vitro assays also demonstrated that miR-34a suppressed the proliferation and migration and promoted the apoptosis of HUVECs. These results indicate that miR-34a may accelerate vascular endothelial injury in the development of hypertension.

Hypertension is a complex and common disease resulting from the interaction of environmental and genetic factors, and the molecular mechanism underlying the development of hypertension remains unclear (18). Recent studies demonstrated that numerous miRNAs were involved in the development of hypertension by mediating vascular remodeling and injury of the heart, kidney and other organs $(19,20)$. For instance, miR-122 induced endothelial NO metabolic disorder and disrupted diastolic and contractile function of the vascular endothelium by targeting solute carrier family 7 member 1 , leading to the development of primary hypertension (21). Furthermore, miR-204 may be involved in vascular remodeling by regulating the proliferation and apoptosis of vascular smooth muscle cells (22). In addition, a number of other miRNAs have been associated with hypertension, including miR-296-5p, let-7e, miR-15b and miR-185, and their roles in regulating the development of hypertension require further investigation (23). The results of the present study demonstrated that miR-34a was significantly upregulated in patients with phase III hypertension compared with patients presenting with phase I and II hypertension, thus indicating that miR-34a may be closely associated with hypertension.

The vascular endothelium, which envelops circulating blood in a continuous monolayer of squamous cells, serves key functions in the regulation of blood flow and exchange of water and small molecules (24). Additionally, it has been observed that continuous hypertension may induce vascular endothelial injury and vascular endothelial dysfunction (25). Numerous miRNAs have been implicated in hypertension-induced vascular endothelial injury (26). miR-34a is a recently identified miRNA that has been associated with tumor development, vascular injury, cell proliferation and apoptosis (27). Furthermore, overexpression of miR-34a may inhibit the proliferation and migration of various tumor cells, including lung, colon and gastric cancer cells $(28,29)$. Overexpression of miR-34a may also induce the apoptosis of human brain glioblastoma cells (30). In particular, miR-34a has been closely associated with pulmonary arterial hypertension, cell proliferation and apoptosis in patients with chronic obstructive pulmonary disease (31). The present study demonstrated that miR-34a may suppress the proliferation and migration and promote the apoptosis of HUVECs, indicating a correlation between miR-34a upregulation and vascular endothelial injury.

Using bioinformatics prediction methods, Tgif2 was identified as a potential target of miR-34a. Tgif 2 is a member of the three-amino-acid loop super family, and the TGIF2 protein encoded by Tgif 2 mRNA may bind to Smad to inhibit the transforming growth factor- $\beta$ signaling pathway (32). Additionally, members of the TGIF family have been documented to promote cell proliferation and differentiation and inhibit cell apoptosis (33). However, there is no reports in the role of TGIF in hypertension. The present study revealed that miR-34a may directly bind to the 3'UTR of Tgif 2 mRNA. Therefore it was speculated that miR-34a suppressed the proliferation and migration and promoted the apoptosis of HUVECs by downregulating Tgif 2 expression and aggravating vascular endothelial injury. 
In summary, miR-34a was upregulated in the peripheral blood of patients with hypertension, and overexpression of miR-34a may have promoted vascular endothelial injury through targeting of Tgif2. Therefore, miR-34a may be a potential marker for the clinical diagnosis and treatment of primary hypertension and vascular injury.

\section{Acknowledgements}

The present study was supported by the National Natural Science Foundation of China (grant no. 81372473) and the China Postdoctoral Science Foundation (grant no. 2014M550766).

\section{References}

1. Bönner G: Use of behavioral therapies in hypertention. MMW Fortschr Med 157: 63-64, 2015.

2. Iaitskiǔ NA, Bedrov AIa, Maslevtsov DV, Tsvetkova EA and Moiseev AA: Ischemic heart disease and arterial hypertention as risk factors of surgical treatment of patients with infrarenal segment of aortic aneurysm. Vestn Khir Im I I Grek 172: 11-15, 2013.

3. Leopold JA: Catheter-based therapies for patients with medication-refractory pulmonary arterial hypertension. Circ Cardiovasc Interv 8: e003332, 2015.

4. Xu H, Zheng H, Huang J, Shen Y and Luo M: T-cell subsets are associated with serum homocysteine concentration in patients with essential hypertension. Clin Exp Hypertens 39: 377-381, 2017.

5. Tang S, Zeng W, Qin J and Jiang M: Diagnosis and treatment of portal hypertension caused by superior mesenteric arteriovenous fistula. Zhonghua Gan Zang Bing Za Zhi 23: 638-640, 2015 (In Chinese)

6. Balduino Mendes AB, Giollo-Junior LT, de Andrade DO, Gregório ML, Yugar-Toledo JC and Vilela-Martin JF: How to investigate the vascular changes in resistant hypertension. Curr Hypertens Rev 12: 139-147, 2016.

7. Jenkins D: Pulmonary endarterectomy: The potentially curative treatment for patients with chronic thromboembolic pulmonary hypertension. Eur Respir Rev 24: 263-271, 2015.

8. Vaillancourt M, Ruffenach G, Meloche J and Bonnet S: Adaptation and remodelling of the pulmonary circulation in pulmonary hypertension. Can J Cardiol 31: 407-415, 2015.

9. Caillon A, Mian MOR, Fraulob-Aquino JC, Huo KG, Barhoumi T, Ouerd S, Sinnaeve PR, Paradis P and Schiffrin EL: $\gamma \delta$ T cells mediate angiotensin II-induced hypertension and vascular injury. Circulation 135: 2155-2162, 2017.

10. Wang X, Yang Y, Yang D, Tong G, Lv S, Lin X, Chen C and Dong W: Tetrandrine prevents monocrotaline-induced pulmonary arterial hypertension in rats through regulation of the protein expression of inducible nitric oxide synthase and cyclic guanosine monophosphate-dependent protein kinase type 1 . J Vasc Surg 64: 1468-1477, 2016.

11. Cao Y, Jiang Z, Zeng Z, Liu Y, Gu Y, Ji Y, Zhao Y and Li Y: $\mathrm{Bcl}-2$ silencing attenuates hypoxia-induced apoptosis resistance in pulmonary microvascular endothelial cells. Apoptosis 21: 69-84, 2016.

12. Matkovich SJ, Dorn GW II, Grossenheider TC and Hecker PA: Cardiac disease status dictates functional mRNA targeting profiles of individual microRNAs. Circ Cardiovasc Genet 8: 774-784, 2015.

13. Kriegel AJ, Baker MA, Liu Y, Liu P, Cowley AW Jr and Liang M: Endogenous microRNAs in human microvascular endothelial cells regulate mRNAs encoded by hypertension-related genes. Hypertension 66: 793-799, 2015.

14. Lorenzen JM: Vascular and circulating microRNAs in renal ischaemia-reperfusion injury. J Physiol 593: 1777-1784, 2015.

15. Wang MM, Fang MX, Chen LG, Wang HQ, Liu HJ and Tang HL: Differential expression of microRNA in endothelial cells incubated with serum of hypertension patients with blood-stasis syndrome. Chin J Integr Med 21: 817-822, 2015.
16. Wang J, Hong Z, Wu L, Ding B, Bi Y, Gu Z and Li W: Dietary intake and cardiometabolic biomarkers in relation to insulin resistance and hypertension in a middle-aged and elderly population in beijing, China. Appl Physiol Nutr Metab 42: 869-875, 2017.

17. Livak KJ and Schmittgen TD: Analysis of relative gene expression data using real-time quantitative PCR and the 2(-Delta Delta C(T)) method. Methods 25: 402-408, 2001.

18. DeCicco D, Zhu H, Brureau A, Schwaber JS and Vadigepalli R: MicroRNA network changes in the brain stem underlie the development of hypertension. Physiol Genomics 47: 388-399, 2015.

19. Cengiz M, Yavuzer S, Kılıçkıran Avcı B, Yürüyen M, Yavuzer H, Dikici SA, Karataș ÖF, Özen M, Uzun H and Öngen Z: Circulating miR-21 and eNOS in subclinical atherosclerosis in patients with hypertension. Clin Exp Hypertens 37: 643-649, 2015.

20. Qi H, Liu Z, Liu B, Cao H, Sun W, Yan Y and Zhang L: micro-RNA screening and prediction model construction for diagnosis of salt-sensitive essential hypertension. Medicine (Baltimore) 96: e6417, 2017.

21. Yang Z and Kaye DM: Mechanistic insights into the link between a polymorphism of the 3'UTR of the SLC7A1 gene and hypertension. Hum Mutat 30: 328-333, 2009.

22. Potus F, Graydon C, Provencher S and Bonnet S: Vascular remodeling process in pulmonary arterial hypertension, with focus on miR-204 and miR-126 (2013 Grover Conference series). Pulm Circ 4: 175-184, 2014.

23. Bockmeyer CL, Maegel L, Janciauskiene S, Rische J, Lehmann U, Maus UA, Nickel N, Haverich A, Hoeper MM, Golpon HA, et al: Plexiform vasculopathy of severe pulmonary arterial hypertension and microRNA expression. J Heart Lung Transplant 31: 764-772, 2012.

24. Zhang L, Wang X, Miao Y, Chen Z, Qiang P, Cui L, Jing H and Guo Y: Magnetic ferroferric oxide nanoparticles induce vascular endothelial cell dysfunction and inflammation by disturbing autophagy. J Hazard Mater 304: 186-195, 2015.

25. Renga B, Cipriani S, Carino A, Simonetti M, Zampella A and Fiorucci S: Reversal of endothelial dysfunction by GPBAR1 agonism in portal hypertension involves a AKT/FOXOA1 dependent regulation of $\mathrm{H} 2 \mathrm{~S}$ generation and endothelin-1. PLoS One 10: $\mathrm{e} 0141082,2015$

26. Deng L, Blanco FJ, Stevens H, Lu R, Caudrillier A, McBride M, McClure JD, Grant J, Thomas M, Frid M, et al: MicroRNA-143 activation regulates smooth muscle and endothelial cell crosstalk in pulmonary arterial hypertension. Circ Res 117: 870-883, 2015.

27. Ye Z, Fang J, Dai S, Wang Y, Fu Z, Feng W, Wei Q and Huang P: MicroRNA-34a induces a senescence-like change via the down-regulation of SIRT1 and up-regulation of $\mathrm{p} 53$ protein in human esophageal squamous cancer cells with a wild-type p53 gene background. Cancer Lett 370: 216-221, 2016.

28. Yang $\mathrm{Hu}$, Qingha Pu, Bin Cui and Jia Lin: MicroRNA-34a inhibits tumor invasion and metastasis in gastric cancer by targeting Tgif2. Int J Clin Exp Pathol 8: 8921-8928, 2015.

29. Wang H, Zhao X, Guo C, Ren D, Zhao Y, Xiao W and Jiao W: Aptamer-dendrimer bioconjugates for targeted delivery of miR-34a expressing plasmid and antitumor effects in non-small cell lung cancer cells. PLoS One 10: e0139136, 2015.

30. Li SZ, Hu YY, Zhao J, Zhao YB, Sun JD, Yang YF, Ji CC, Liu ZB, Cao WD and Qu Y: MicroRNA-34a induces apoptosis in the human glioma cell line, A172, through enhanced ROS production and NOX2 expression. Biochem Biophys Res Commun 444: 6-12, 2014.

31. Mizuno S, Bogaard HJ, Gomez-Arroyo J, Alhussaini A, Kraskauskas D, Cool CD and Voelkel NF: MicroRNA-199a-5p is associated with hypoxia-inducible factor-1 $\alpha$ expression in lungs from patients with COPD. Chest 142: 663-672, 2012.

32. Willer A, Jakobsen JS, Ohlsson E, Rapin N, Waage J, Billing M, Bullinger L, Karlsson S and Porse BT: TGIF1 is a negative regulator of MLL-rearranged acute myeloid leukemia. Leukemia 29: 1018-1031, 2015.

33. Powers SE, Taniguchi K, Yen W, Melhuish TA, Shen J, Walsh CA, Sutherland AE and Wotton D: Tgif1 and Tgif2 regulate nodal signaling and are required for gastrulation. Development 137: 249-259, 2010. 